\title{
Unit Nonresponse Errors in Income Surveys: A Case Study
}

\author{
MICHELE LALLA*, DAVIDE FERRARI, PATRIZIO FREDERIC \\ Department of Political Economy, University of Modena and Reggio Emilia, Via Jacopo \\ Berengario 51 - 41121 Modena, Italy. (*Author for correspondence) E-mail: \\ michele.lalla@unimore.it
}

\begin{abstract}
A survey on the economic and social conditions of households in the city of Modena was carried out in 2002 and in 2006 (two waves) by the CAPP (Centre for Analyses of Public Policies). In first wave of 2002, each designated sampling unit (i.e., the family) had three units as reserves. If the first refused to be interviewed, the interviewer contacted the three reserves, one after the other, until obtaining either one respondent or four non-participant units. At the end of the survey four categories of units were distinguished: interviewees, refusals, noncontacts, and unused reserves. All units were matched with their corresponding record in the databases of the Ministry of Finance of 2002 and the Census of 2001. The resulting data set permitted the analysis of unit or total nonresponses. The distribution of fiscal income showed different shapes for the four categories, implying a selective participation of the families. The interviewees yielded a positive bias of about $600 €$, holding constant other factors. The selection of the significant factors affecting nonresponse was performed via backward elimination in a logit model and with the lasso method. Participation increased as fiscal income and age increased and by education level (secondary school and university degree), while it decreased among entrepreneurs, independent workers, managers, and medium-to-low skilled workers.
\end{abstract}

Key words: fiscal income, reserve units, response bias, logit model, lasso method

\section{Introduction}

The phases of sample selection and data collection encounter some specific difficulties concerning the sources of errors, which are termed nonsampling errors because they do not depend on a random selection process of the sampling units or design. Nonsampling errors are usually classified into three categories (Lessler and Kalsbeek 1992; Särndal et 
al. 1992): (i) Frame errors or undercoverage refer to the failure of the frame to give access to all elements that belong to the target population; (ii) Nonresponse errors involve the failure to obtain information on the units selected for the sample. It assumes two forms. Unit or total nonresponse happens when the entire interview or questionnaire is missing due to refusal, or due to untraceability, not being at home, incapacity to answer, and so on. Item or partial nonresponse occurs when an individual fails to answer only one or a few questions or items; (iii) Observation errors occur when a sampling unit is selected and observed, but the final recorded value for that unit differs from the true value. These errors are distinguished in two groups. One group includes measurement errors, which may arise in the data collection phase and involve the interviewer, the respondent, the questionnaire, and the interview mode. The other group consists of processing errors, which arise in the data processing phase and involve coding, transcription, imputation, editing, outlier treatment, and so on. Only the total nonresponse error category will be examined in the following.

Once the sample is drawn, standard estimation procedures on collected data provide unbiased values only when a response rate of $100 \%$ is attained. Therefore, the reduction of nonresponse errors represents an important challenge in survey research, as the primary objective of the latter is to obtain the highest possible response rate, while in practice it is almost impossible to obtain the participation of every selected statistical unit in a large sample, regardless of the method used for data collection. Actually, it is common practice to consider the response rate as an indicator of survey quality and a high rate, especially for income data, can entail a considerable amount of human effort and financial resources, even to achieve a participation rate of $40 \%$ of all units originally selected for the sample (Goyder 1987; Levy and Lemeshow 1991). This happens even when a survey protocol includes many telephone calls to make an appointment with at least one person in the household sampling unit or to revisit households in which the person to be interviewed was not home at the time of the first visit, and when the protocol calls for telephone follow-ups or for subsequent mailings. For example, the Survey of Household Income and Wealth (SHIW), carried out every two years by the Bank of Italy (2008), achieved in 2006 a response rate of $30.2 \%$ in the cross-sectional component. The response rates of the surveys carried out in the International Social Survey Program (ISSP) for the Federal Republic of Germany varied over time (1999-2005) and region (west, east), ranging from $34.7 \%$ to $51.8 \%$ (Hüfken 2009). The mail business survey carried out in Italy by Istat presented a response rate ranging from $40 \%$ to $53 \%$ in the 1999-2004 period (Brancato et al. 2006). In 1994, the European Community Household Panel (ECHP) presented response rates varying by country (Gallo et al. 2004): Luxemburg (35.8\%), Germany (47.8\%), Ireland (55.8\%), Denmark (63.3\%), Italy (89.1\%), Spain and Greece (90.1\%); but ECHP was mandatory which represented the 
strongest predictor of response (de Leeuw and de Heer 2002). The Labour Force Survey, which was also mandatory, showed high response rates, also varying by country, and always higher than 70\% (de Heer 1999).

The objective of this paper is to analyze the effect of item nonresponse errors in a survey on household economic and social conditions in the Province of Modena ICESMO, acronym for Indagine sulle Condizioni Economiche e Sociali nella provincia di MOdena - carried out in 2002 by the CAPP (Centre for Analyses of Public Policies) of the University of Modena's Department of Political Economy. The probability of nonparticipation in the survey was assumed to be high, given that the SHIW participation rate generally ranged between $30 \%$ and $40 \%$ over time, in its cross-sectional component. Therefore, for each sampling unit (the family) drawn, three supplementary units were selected as reserves. If the first unit refused to be interviewed or was definitely considered to be untraceable, the interviewer would contact the next unit on the list of three reserves, and so on. The process could stop either because one unit on the list was interviewed or because all four units were contacted and all refused to be interviewed (or some or all were untraceable). At the end of the survey, four categories or "samples" of selected units were obtained: respondents or interviewees, refusals, noncontacts or untraceable units, and unused reserves - hereinafter referred to as the "types of samples", i.e., a variable which in the data set assumed the values $1,2,3$, and 4 to denote, respectively, these categories or samples.

The fiscal database of the Ministry of Finance of 2002 became available after the survey was conducted; this was an unexpected event as the archives are strictly protected by privacy policies. This availability allowed for the exact matching of the sampling units, using their fiscal identity numbers, with the corresponding records in the databases of the Ministry of Finance of 2002 and the Census of 2001, respecting anonymity. Therefore, the income declared to fiscal authorities - hereinafter referred to as Fiscal Income (FI) - was available for all units required to file a declaration, i.e., for all taxpayers. The analysis of FI with respect to some available factors could shed light on the bias of the income estimates and on the different behaviours of those groups showing different attitudes towards participation in a survey, even if the fiscal data are affected by evasion, elusion, and erosion. However, it was not possible to plan the study with this possibility in mind, as access to the fiscal database was precluded at that time. Therefore, the procedures carried out here and the results obtained are similar to a post-hoc analysis. Nevertheless, this study comparing the income data of respondents and nonrespondents is the first ever conducted in Italy on a large scale and a rarity in the literature. In fact, many validation studies deal with item nonresponse and measurement error in earning survey data (among others, Moore et al. 2000; Bound et al. 2001; Shräpler 2006), but also in many other types of survey data collected through interviews: on medical expenditure 
(Cohen and Carlson 1995), on firms and consumers for industrial or marketing research (de Bruwer 1995; Delener 1995; Collier 2007), on sensitive issues (Kupek 1998; Tu and Liao 2007), and so on. Also techniques used to handle item nonresponse have been developed in articles (among others, Franses et al. 1999; Stocké 2006) and books (Little and Rubin 1987; Rubin 1988; Särndal and Lundström 2005). However, studies on unit nonresponse are very rare because in the first wave, the refusals and noncontacts are completely unknown, i.e., it is almost impossible to have information on them for an understanding of the mechanism inducing their behaviour or the factor determining their failure to participate. Therefore, analyses of unit nonresponse are prevailingly carried out on longitudinal survey data, starting at least from the second wave, because in the waves subsequent to the first, the data collected in the previous waves or the first wave are available for the individuals now refusing the interview or who are untraceable (Cannari and D'Alessio 1992; Campanelli and O'Muircheartaigh 1999).

In the data set obtained through the procedure described above, the variables extracted from the fiscal database (gender, age, fiscal income, etc.) and the census database (gender, age, education level, occupation, etc.) were available for each selected individual: interviewee, refusal, noncontact or unused reserve. Therefore, this represented a rare opportunity to verify selectivity bias for FI in the sample of interviewees and to analyse the unit nonresponse error with respect to basic variables in the income survey carried out in the first wave or one-shot. Although the results cannot be generalised to the whole country due to the limited geographical study area, they do suggest interesting directions for further study.

The paper is organised as follows. Section 2 discusses some problems related to nonresponse and measurement errors in survey data and briefly reviews the main results of other surveys. Section 3 describes the main characteristics of the ICESMO survey and of the fiscal data, as well as the basic features of the sample types. Section 4 illustrates, separately, the effect of nonresponse on the estimation of FI and the determining factors regarding response or nonresponse of taxpayers. Section 5 presents the determinants of response obtained via the lasso method, which also permits easy identification of the interaction between the determinants. Section 6 concludes with some comments.

\section{Nonresponse and measurement errors in surveys on income}

Surveys on income, private property, and savings are complex and subject to various sources of error (Curtin et al. 1989; Quintano and Lucev 1990; Juster and Smith 1997; Hurd et al. 2003). Interviewer training is essential, in order to persuade the selected sampling units to participate in the survey and to collect high quality data (Couper and 
Groves 1992; Hox and de Leeuw 2002). However, often training is not enough because the data collection process is subject to time and logistic limits, as well to constraints relating to human and financial resources, which are generally available on the basis of fixed budgets and affect the planning of the sampling design and the strategies adopted to gather high quality data. Therefore, the nonsampling errors are physiological traits of each concrete sampling design, particularly in surveys on income, and they can depend on many factors. For example, the analyses of the attrition in the panel sub-sample of the SHIW showed that nonresponses were more frequent for households living in urban areas and those residing in the North, that the participation rates declined as income increased and household size decreased, and that the age of the head of the household affected the reliability of responses in a more complex way (Cannari and D'Alessio 1992). The investigation of the impact of unit nonresponse on the estimation of income and wealth in the SHIW proved that nonresponses were more frequent among wealthier households, which will affect the concentration of income and wealth (D'Alessio and Faiella 2002).

Several studies have analysed nonresponse in a large number of surveys, prevailingly in terms of social phenomena, and, although general conclusions are difficult to formulate, the studies indicate that nonresponse is not dependent on individual characteristics, such as age, education level, and social status (Biemer and Lyberg 2003). There are also many theories of survey participation. Each explains the behaviours of interviewees in a given framework and provides strategies and suggestions to increase response rates. Knowledge of the characteristics of nonrespondents could be useful for the evaluation of the quality of a survey and the representativeness of a sample, for an understanding of the process leading from the sample selected to the sample observed and, perhaps, for quantifying the bias attributable to nonresponse. This approach will be adopted here to analyse the surveyed income data.

Item nonresponse errors could partially overlap measurement errors because the former could originate from an ambiguous or inadequate statement. Therefore, their classification is based on the generating cause, which consist of (1) Instrument errors are referable to the questionnaire and include improperly formulated questions, bad arrangement of the items, unadjusted battery, and so on. (2) Technique errors derive from the methodology and the strategies used for data collection, i.e., the interview mode, be it by telephone, mailing, personal interview, self-administered questionnaire or another medium, with or without computer-assisted data entry technology. (3) Interviewer errors are due to causes or factors within the dynamics of the interview, depending on the personal characteristics of the interviewer (gender, age, education level, voice quality, attire) and on interaction modalities that affect the interviewees, thus encouraging or discouraging them to participate in the survey and whether or not to respond to an item.

(4) Errors of the interviewees or respondents, like those of interviewers, are due to causes 
within the dynamics of the interview and they depend on their personal characteristics (gender, age, education level, personality), their comprehension or recollection of past events, whether they are qualified to answer and willing to be truthful, as well as the conditions created during the interview (see, among others, Tourangeau et al. 2000; Moore et al. 2000; Biancotti et al. 2004).

The analysis of data affected by measurement errors presents major obstacles when the errors have a specific and generally unknown pattern, while assumptions on these patterns are often suggested by accommodation rather than persuasion, simplification rather than representation (Bound et al. 2001). However, this kind of nonresponse error will not be considered in the following.

\section{Data sources}

\subsection{SURVEY ON ECONOMIC AND SOCIAL CONDITIONS OF HOUSEHOLDS IN MODENA}

The survey on economic and social conditions of households in the Province of Modena was carried out in 2002 by the CAPP (Centre for Analyses of Public Policies) of the University of Modena's Department of Political Economy and was based on two-stage cluster sampling, stratified with the respect to the geographic area subdivided in accordance with the socio-healthcare districts, in which the municipalities were the primary sampling units and households were the secondary sampling units. The municipalities were distinguished in two categories, as in other surveys of this kind (Banca d'Italia 2008): self-representing (SR) municipalities were the chief towns in the districts, while all the others were non-self-representing (NSR) municipalities. The number of NSR municipalities per district was proportional to the number of resident households: one NSR unit each 10000 households. Firstly, the Provincial sample size was subdivided by the districts proportionally to their number of resident households, thus obtaining the district sample size. Secondly, the district sample size was subdivided by the municipalities selected in the sample proportionally to their number of resident households, thus obtaining the municipality sample size. There were 789 households interviewed in the Province of Modena, not including Modena, but their inclusion in the matching with fiscal data was not possible because the fiscal databases of their municipalities were not available, nor were the fiscal codes of the family members.

The sample selected in the municipality of Modena provided a precision in the estimates that was almost equal to that of the rest of Province. It was stratified by the size of the family $(1,2,3,4$ or more members), by the age of the head of the household (up to 34 years, 35-49 years, 50-64 years, 65-74 years, 75 years or more), and by gender. The 
sample sizes in the strata were obtained by proportional allocation. The households interviewed in Modena amounted to 589 with 1388 subjects out of a target of 637 households (Baldini et al. 2004). Therefore, considering three reserves per sampling unit, the total number of selected households was 637 times four, equal to 2548 units, as indicated in Table I, which also shows the percentages of interviewees, refusals, noncontacts, and unused reserves, as well the corresponding SHIW data (Banca d'Italia 2002).

Table I. Absolute frequencies and percentages regarding the surveys

\begin{tabular}{lrrrrrr}
\hline Type of sample & \multicolumn{2}{c}{ ICESMO 2002 } & SHIW 2002: non panel & \multicolumn{2}{r}{ SHIW 2002: panel } \\
\cline { 2 - 7 } & $n$ & $\%$ & $n$ & $\%$ & $n$ & $\%$ \\
\hline Interviewees & 589 & 33.4 & 4128 & 27.5 & 3873 & 65.8 \\
Refusals & 704 & 39.9 & 8583 & 57.2 & 1878 & 31.9 \\
Noncontacts & 472 & 26.7 & 2287 & 15.2 & 133 & 2.3 \\
Total of used units & 1765 & 100.0 & 14998 & 100.0 & 5884 & 100.0 \\
Unused reserves & 783 & 30.7 & & & & \\
Ineligible* & & & 570 & 3.8 & 232 & 3.9 \\
\hline Total & 2548 & & 15568 & & 6116 &
\end{tabular}

* Nonexistent households (Tax Register address no longer valid due to death, change of address or incorrect address). In the ICESMO 2002 survey, the ineligible sample was included in the noncontact sample.

The questionnaire used in the interview requested information about many variables and, specifically, those concerning net earnings, real and personal estate, capital and financial assets over the last twelve months. The interview period was from June 2002 to December 2002. Therefore, the beginning of the reference income period varied from June 2001 to December 2001. Such a mobile reference income period, differing among the subjects, represented the most critical aspect for at least two reasons. First of all, hypothetical and nontrivial operations were necessary to obtain the individual yearly surveyed income (SI) to be compared with FI, but a detailed discussion of these operations and results is beyond the scope of the paper. Secondly, there is some evidence that recall period length contributes to faulty recall (Moore et al. 2000). For example, it would have been better to refer to income over an annual period coinciding with the calendar year.

\subsection{MATCHING OF FISCAL AND SURVEY DATA}

The fiscal database of the city of Modena referred to the FIs of 2002 of all taxpayers in the city. It was obtained unexpectedly by its statistics office, which selected the ICESMO sampling units. In fact, ordinarily, the office in charge could examine only one taxpayer 
at a time, through a system implemented by the Ministry of Finance for access to the tax register on the part of local authorities (SIATEL: Sistema di Interscambio dell'Anagrafe Tributaria degli Enti Locali), without access to the entire database under its jurisdiction. In any case, the fiscal database of 2002 was matched with the census database of 2001. The resulting file was matched with all the sampling units included in the survey design and four samples were thus obtained: interviewees, refusals, noncontacts or untraceable units, and unused reserves. The total size of the overall sample included 6010 individuals, but 84 individuals were missing from the fiscal database, thus reducing the overall sample size to 5926.

The objective of our analysis is to assess the effect of nonresponse error on the estimation of income and, specifically, on FI. Therefore, the individuals who were not required to file an income tax return should be eliminated from the four samples. This was done by eliminating all individuals who had not filed a tax form (TF), i.e., entire tax form missing. The types of tax forms utilized for reporting FI were: the TF-730, TFUnico, and TF-770. The TF-730 and TF-Unico, alternatively filed by the taxpayer (the first is more simplified and utilized by the majority of labour dependents), contain all the details regarding sources of income (land, buildings, estate, employment, other, total, and taxable income), tax deductions and tax allowances (for wife, children, relatives, pension, and others) that are relevant for the application of the personal income tax (IRPEF). The TF-770, which regards employer income, also contains information on the taxable income of dependents who are exempted from filing a tax declaration (as all the relevant information is provided by the employer). There were 1530 individuals without tax forms, i.e., 1446 plus 84 unmatched individuals (Table II). Therefore, the final number of processed forms was equal to 4480, of which 7 forms filed by individuals declaring an income equal to 0 . Moreover, note that the type of tax form was associated with occupation category; in fact, the TF-Unico showed a higher mean declared income than the other types of tax forms because it is used prevailingly by managers, high public officials, non-standard workers, professional persons, the self-employed, entrepreneurs, and managers. These individuals were very hesitant about being interviewed about their income and distrustful of interviewers asking for reserved information about sources and amounts of income.

Referring to Table II and those that follow, with data on the type of sample in the columns, the comments are prevailingly based on the comparisons of the FI mean in a cell with the "corresponding marginal total of the row", which will be abbreviated by the expression "row total". The "total mean FI" denotes the row total of totals. The sample of interviewed individuals significantly overestimated the total mean FI by $8.4 \%(p<0.021)$, but this was significant only for the TF-730 group. In the sample of refusals, the FI mean was lower than the total mean FI by $5.0 \%$, but the difference proved to be significant only 
at the level of $1 \%$ in the TF-730 sub-sample. However, the TF-Unico sub-sample showed an FI mean greater than its row total mean by $11.0 \%$, but it did not differ significantly from zero. In the sample of noncontacts, the FI mean was lower than the total mean FI by $5.0 \%$, but it was not significantly different from zero. In the TF-Unico sub-sample the difference with its row total was $-10.4 \%$, but it proved to be statistically equal to zero. The sample of unused reserves did not produce any statistically significant differences for FI means.

The FI mean for women proved to be lower than that for men and it also differed between the sub-samples determined by the type of tax form. In addition, there was a significant interaction between type of tax form and gender. However, for the sake of brevity, only gender by type of sample is reported in Table III. FI means proved to differ between the categories of gender and type of sample. The interviewed sample showed a higher mean FI than those of the other samples: 3.8\% for women and 12.3\% for men, with respect to their row total mean FI. The latter was statistically different from zero at the 3\% level of significance. There were no other significant values in the other samples, even if the mean FI for women was lower than its row total by $6.7 \%$ in the sample of refusals and the mean FI for men was lower than its row total by $8.0 \%$ in the sample of noncontacts. 
Table II. Descriptive statistics on fiscal income in 2002 by type of tax form and by type of sample

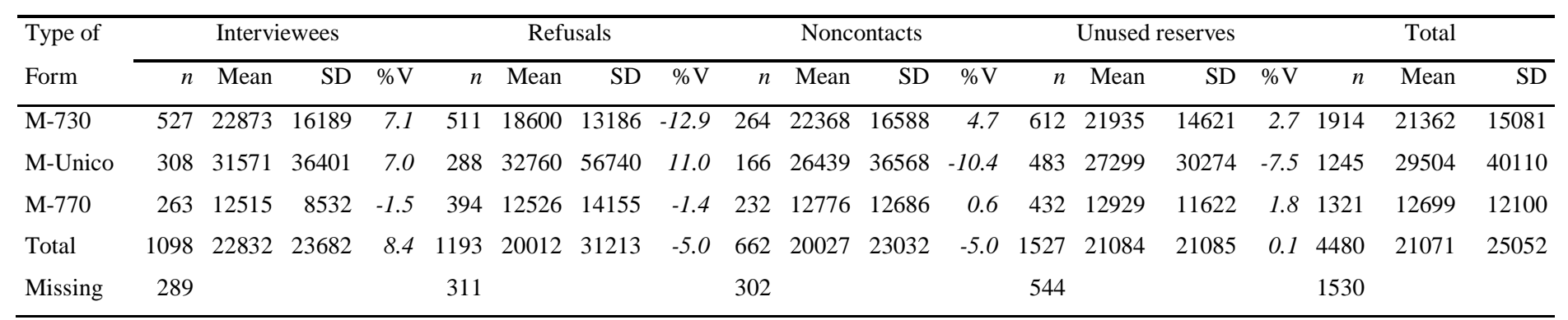

Legend: \%V = percentage variation of the mean $=100$ (mean - row total mean)/ (row total mean)

Table III. Descriptive statistics on fiscal income in 2002 by gender and by type of sample

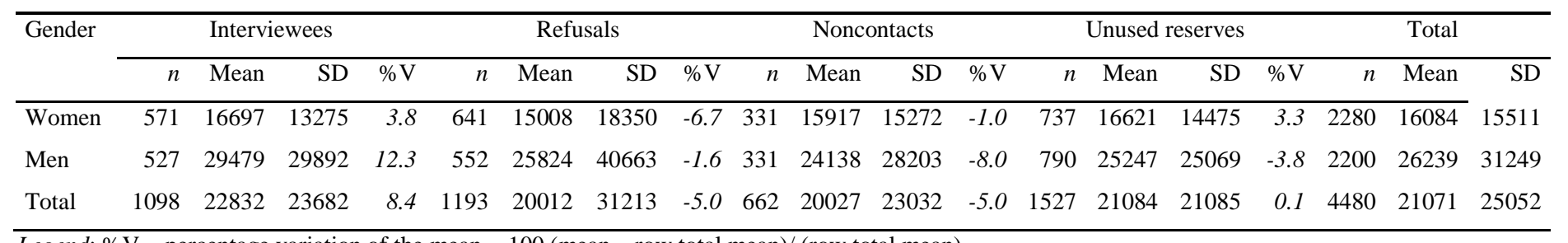

Legend: \% V = percentage variation of the mean $=100$ (mean - row total mean)/ (row total mean) 


\section{Factors affecting nonresponse errors}

This analysis can be carried out assuming that the statistical unit is either the household, whose ideal representative unit is its head, or the single individual. On the one hand, the analysis carried out at the household level does not provide a realistic representation of the dynamics of the interview because often the person contacted by the interviewer and refusing the interview is not the head of the household, but another cohabitant, usually the spouse. Therefore, the statement: «Nonresponse rate is affected by the age and the education level of the head of the household» may represent only an abstract data set description. On the other hand, the analysis carried out at the individual level is not a realistic representation either, for only one member of the household is making the decision whether to participate or not. Therefore, once again, the statement: «Nonresponse rate is affected by the age and the education level of the individual» may represent only an unreal data description. In this case, the structure of the household is lost for those who were not interviewed and the individual level of analysis is a forced choice.

The characteristics of sample are summarised in Figure 1 and in four tables reporting the sampling sizes and the FI means, disaggregated by the type of sample with respect to the deciles of FI, age, education level, and occupation. The interviewees revealed only one case in which FI=0. Among the refusals there was one negative value (the lowest, with $\mathrm{FI}=-37760 €$ ) and there were three cases in which $\mathrm{FI}=0$. Among the noncontacts there were three negative values and one case with $\mathrm{FI}=0$, whereas the unused reserves displayed three negative FI values and two cases in which FI $=0$. The density distributions showed long heavy right tails, especially for refusals, whose right tails exceeded 300,000€ reaching 500,000€. Therefore, Figure 1 shows the density distributions, one for each sample, of the positive FIs, ranging from zero to $100,000 €$ in order to provide a readable graph. In general, income distribution appeared to vary significantly across the samples. Table IV illustrates the numerical values of the density distributions of FI grouped by deciles, as is usual in income analyses. It should be noted that an FI of 5,104€ reached a very high absolute frequency in the sample of interviewees and the sample of refusals, involving a difference in absolute frequencies between the first and the second decile. 


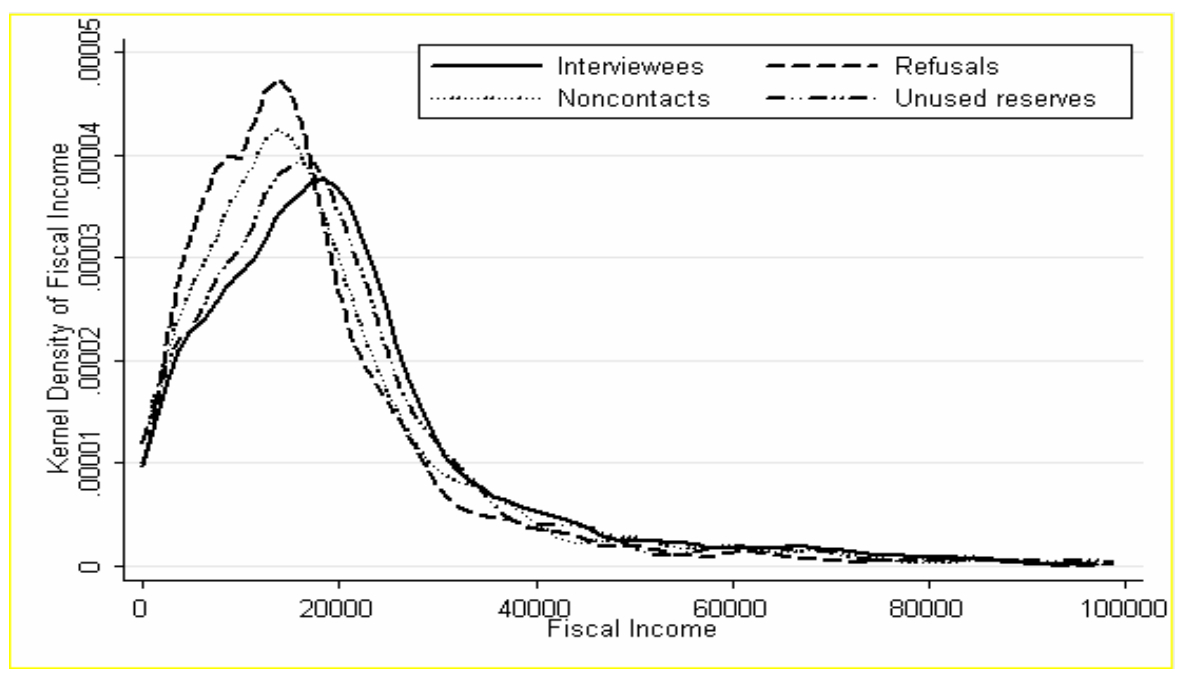

Figure 1. Epanechnikov kernel density estimates of fiscal income in 2002 for first-wave interviewees, refusals, noncontacts, and unused reserves

Table V displays the joint distribution of age (subdivided into classes) and type of sample. The FI means showed a nonlinear parabolic trend where the highest mean was in the 50 to 59-year-old age group and the highest curve was observed for the interviewees. The profiles of the means for the different types of samples tended to differ from that of the row marginal distribution and there was no interaction between the age classes and the type of sample. In the sample of interviewees, three age classes showed an overestimation of the row total mean FI: the 30 to 39 -year-old, the 50 to 59-year-old, and the «greater than or equal to 70-» year-old age classes. Only in the last case was the mean statistically greater than that of its row total mean, at a $5 \%$ level of significance, and in the one-tailed test, the percentage variation was $24.4 \%$. In the sample of refusals, the age classes under fifty years showed FI means greater than their row total means, while those over fifty years of age had FI means lower than their row total means. However, there were not any significant differences with respect to their row total means, even if the age classes «under 30» and 40 to 49 years of age showed overestimations of $12.4 \%$ and $13.8 \%$ of their row total means, respectively. In the sample of noncontacts, two age classes presented FI means differing from their row total means: the 30 to 39-year-old age class with a percentage variation equal to $-15.8 \%$, which was significant at the $1 \%$ level, and the «greater than or equal to 70» age class with a percentage variation equal to $-10.7 \%$, which was significant at the $5 \%$ level in a one-tailed test. In the sample of unused reserves, the FI means did not reveal any differences with respect to age. Note that unless indicated otherwise, the significance level always refers to a two-tailed test. Moreover, note that these comparisons, as the others carried out below, were affected by a remarkable heterogeneity of FI between classes or sub-groups, implying that it could happen that a variation lower than others, but with a lower standard deviation, could be 
statistically different from zero, while the others are not. The number of cases in the category could also affect the results.

Education level by the type of sample (Table VI) showed that FI means increased as the education level increased. The highest FI mean was observed among refusals and noncontacts, while the missing "education level" data category showed means almost equal to those with the lowest education level. The FI means of the education levels in the sample of interviewees did not reveal any significant differences with respect to their row total means, even if the percentage variation for individuals with university degrees was $-10.3 \%$ and for missing data category regarding the education level, it reached $34.7 \%$. In the sample of refusals, individuals with university degrees showed an FI mean greater than the row total mean by $29.3 \%$, which proved to be statistically different from zero, at a $5 \%$ level of significance in a one-tailed test. In the sample of noncontacts, the missing data category regarding the education level showed an underestimation of the row total mean by $30.8 \%$, with a $3 \%$ level of significance. In the sample of unused reserves, no statistical differences were found with respect to the row total marginal distribution.

Three original variables described the position of an individual with respect to the employment: activity status (employed, unemployed, retiree, housewife, etc.), employment status (dependent worker, entrepreneur, self-employed, etc.), and type of job with ten categories. They were regrouped into a single variable, termed simply "occupation". In the grouping, the workers were distinguished between dependent (employed) and independent (self-employed). Subsequently, they were grouped in accordance with the traditional distinction of highly-skilled and medium-to-low-skilled workers or a residual "other" category. The individuals who were not in the labour force were classified as retired workers and "housewives and others". Table VII displays the joint distribution of occupation and type of sample. The four samples showed a common pattern. On the average, the entrepreneurs declared the highest FI compared to others, although it was not exceedingly high. In decreasing order, they were followed by managers, by independent highly-skilled workers, and dependent highly-skilled workers.

The comparisons of cell means with respect to their row total means showed few significant differences. In the sample of the interviewees, the independent other workers and the retirees proved to have means higher than their row total means. The percentages of variations were $40.4 \%(\mathrm{p}<0.059)$ and $16.9 \%(\mathrm{p}<0.013)$, respectively. Therefore, the interview process more easily captured other independent workers and retirees whose incomes were higher than those who refused to be interviewed or were not contacted. Hence, the interviewers should give these categories special attention, along with the category of independent highly-skilled workers. The FI mean of the latter was greater than its row total mean by $12.8 \%$, although it did not significantly differ from zero. In the sample of refusals, the retirees showed an FI mean that was $9.3 \%(\mathrm{p}<0.032)$ lower than 
the row total mean. In the sample of noncontacts, only the missing data category showed an underestimation of the row total mean, by $24.2 \%$, which was significant at a borderline level in a one-tailed test $(\mathrm{p}<0.054)$. In the sample of unused reserves, there were two significant differences. The entrepreneurs showed a negative percentage variation, $-20.1 \%$, which was significant at the $5 \%$ level in a one-tailed test, implying that more frequently they refused to be interviewed or were non-contactable. An underestimation $(-18.7 \%)$ that was significant at the $4 \%$ level in a one-tailed test, was also observed in the "housewife and other" category, implying that in the interview phase their attitude was similar to that of the entrepreneurs. Again, this category merits more attention during the contact phase to succeed in reaching interview acceptance.

The previous tables illustrate the relationships between the dependent variable FI and the single covariates available. These relationships are also more synthetically and appropriately described through a linear regression model, assuming FI, denoted by $Y$, as the dependent variable and the other available information as covariates, $X$. In addition to the variables examined previously, the model also included the type of work schedule (full-time, part-time, missing data), type of payment (paid worker, non-paid worker, missing), the type of contract (long-term contract, short-term contract, missing data), economic sector (industry, commerce, public administration, school, healthcare, services, and "other" including: farming, fishing, construction, and transport), and type of sample (interviewees, refusals, noncontacts, unused reserves). Except for age, the latter are qualitative variables, and were transformed into binary or dummy variables having the value of 1 when they assumed the corresponding modality, and 0 otherwise. Their regression coefficients express the variation of FI with respect to the constant or reference individual: woman, with age equal to zero, Italian middle school education level, housewife and others, type of work schedule missing, type of payment missing, type of contract missing, other economic sector, in the sample of unused reserves. The age of individuals was divided by ten to reduce its range and introduced into the model as a polynomial of the second order to account for the nonlinearity of the relationship. 
Table IV. Maximum and mean fiscal income in 2002 and number of cases by type of sample and by deciles

\begin{tabular}{lrrrrrrrrrrr}
\hline Type of & \multicolumn{10}{c}{ Deciles } \\
sample & $1^{\text {st }}$ & $2^{\text {nd }}$ & $3^{\text {rd }}$ & $4^{\text {th }}$ & $5^{\text {th }}$ & $6^{\text {th }}$ & $7^{\text {th }}$ & $8^{\text {th }}$ & $9^{\text {th }}$ & $10^{\text {th }}$ & Total \\
\hline I. Max & 5104 & 9052 & 12344 & 15337 & 18063 & 20772 & 23702 & 28584 & 42640 & 304574 & 304574 \\
I. Mean & 2841 & 7035 & 10804 & 13892 & 16794 & 19373 & 22029 & 25874 & 34640 & 75957 & 22832 \\
I. $n$ & 123 & 97 & 110 & 110 & 109 & 110 & 110 & 110 & 110 & 109 & 1098 \\
\hline R. Max & 5104 & 6846 & 9925 & 12186 & 14341 & 16363 & 19209 & 24103 & 34771 & 484273 & 484273 \\
R. Mean & 2732 & 5930 & 8457 & 11068 & 13332 & 15275 & 17591 & 21431 & 28097 & 77228 & 20012 \\
R. $n$ & 149 & 90 & 119 & 120 & 119 & 119 & 120 & 119 & 119 & 119 & 1193 \\
\hline N. Max & 4603 & 7465 & 10366 & 13004 & 15323 & 17481 & 20842 & 25482 & 37019 & 301155 & 301155 \\
N. Mean & 1733 & 5867 & 9090 & 11782 & 14046 & 16327 & 19095 & 23118 & 30982 & 68567 & 20027 \\
N. $n$ & 67 & 66 & 66 & 66 & 66 & 67 & 66 & 66 & 66 & 66 & 662 \\
\hline U. Max & 4491 & 8230 & 11300 & 14365 & 16528 & 19331 & 22243 & 27278 & 39578 & 263840 & 263840 \\
U. Mean & 1651 & 6316 & 9870 & 12989 & 15454 & 17945 & 20788 & 24465 & 31981 & 69638 & 21084 \\
U. $n$ & 153 & 153 & 153 & 152 & 153 & 153 & 152 & 153 & 153 & 152 & 1527 \\
\hline
\end{tabular}

Legend I. = Interviewees, R. = Refusals, N. = Noncontacts, U. = Unused reserves, $n=$ no. of cases

Table V. Descriptive statistics on fiscal income in 2002 by age class and by type of sample

\begin{tabular}{|c|c|c|c|c|c|c|c|c|c|c|c|c|c|c|c|c|c|c|c|}
\hline \multirow{2}{*}{$\begin{array}{l}\text { Age } \\
\text { classes }\end{array}$} & \multicolumn{4}{|c|}{ Interviewees } & \multicolumn{4}{|c|}{ Refusals } & \multicolumn{4}{|c|}{ Noncontacts } & \multicolumn{4}{|c|}{ Unused reserves } & \multicolumn{3}{|c|}{ Total } \\
\hline & $n$ & Mean & $\mathrm{SD}$ & $\% \mathrm{~V}$ & $n$ & Mean & SD & $\% \mathrm{~V}$ & $n$ & Mean & SD & $\% \mathrm{~V}$ & $n$ & Mean & SD & $\% \mathrm{~V}$ & $\bar{n}$ & Mean & SD \\
\hline$<30$ & 115 & 11716 & 8416 & -3.2 & 102 & 13610 & 9931 & 12.4 & 80 & 12779 & 10308 & 5.6 & 239 & 11428 & 8445 & -5.6 & 536 & 12106 & 9048 \\
\hline 30-39 & 228 & 23412 & 23989 & 10.9 & 167 & 21444 & 20538 & 1.5 & 167 & 17771 & 13263 & -15.8 & 341 & 21062 & 18902 & -0.3 & 903 & 21118 & 19822 \\
\hline $40-49$ & 198 & 25227 & 19179 & -3.1 & 148 & 29604 & 46108 & 13.8 & 105 & 23858 & 21850 & -8.3 & 301 & 25541 & 21809 & -1.9 & 752 & 26023 & 27788 \\
\hline $50-59$ & 183 & 31041 & 29814 & 8.6 & 124 & 27078 & 44877 & -5.2 & 82 & 28705 & 25604 & 0.5 & 299 & 27652 & 29832 & -3.2 & 688 & 28576 & 32585 \\
\hline $60-69$ & 179 & 21821 & 20646 & -0.5 & 257 & 21346 & 38939 & -2.7 & 97 & 26553 & 43121 & 21.1 & 188 & 20460 & 18477 & -6.7 & 721 & 21934 & 31379 \\
\hline$>=70$ & 195 & 19501 & 26506 & 24.4 & 395 & 14380 & 15577 & -8.3 & 131 & 13995 & 8830 & -10.7 & 159 & 15590 & 11852 & -0.5 & 880 & 15676 & 17465 \\
\hline Total & 1098 & 22832 & 23682 & 8.4 & 1193 & 20012 & 31213 & -5.0 & 662 & 20027 & 23032 & -5.0 & 1527 & 21084 & 21085 & 0.1 & 4480 & 21071 & 25052 \\
\hline
\end{tabular}

$* \% \mathrm{~V}=$ percentage variation of the mean $=100($ mean - row total mean $) /($ row total mean $)$ 
Table VI. Descriptive statistics on fiscal income in 2002 by education level and by type of sample

\begin{tabular}{|c|c|c|c|c|c|c|c|c|c|c|c|c|c|c|c|c|c|c|c|}
\hline \multirow{2}{*}{$\begin{array}{l}\text { Education } \\
\text { level }\end{array}$} & \multicolumn{4}{|c|}{ Interviewees } & \multicolumn{4}{|c|}{ Refusals } & \multicolumn{4}{|c|}{ Noncontacts } & \multicolumn{4}{|c|}{ Unused reserves } & \multicolumn{3}{|c|}{ Total } \\
\hline & $n$ & Mean & SD & $\% \mathrm{~V}$ & $n$ & Mean & SD & $\% \mathrm{~V}$ & $n$ & Mean & $\mathrm{SD}$ & $\% \mathrm{~V}$ & $n$ & Mean & SD & $\% \mathrm{~V}$ & $n$ & Mean & SD \\
\hline Elementary $\mathrm{S}$. & 256 & 13337 & 9532 & 0.2 & 509 & 13307 & 19504 & 0.0 & 202 & 13112 & 9096 & -1.5 & 330 & 13401 & 8622 & 0.7 & 1297 & 13306 & 14096 \\
\hline Middle S. & 230 & 18224 & 16375 & 8.2 & 257 & 16762 & 12530 & -0.5 & 151 & 16417 & 11618 & -2.5 & 423 & 16298 & 12030 & -3.2 & 1061 & 16845 & 13158 \\
\hline Secondary S. & 373 & 23764 & 19744 & 6.9 & 290 & 21003 & 19219 & -5.5 & 204 & 22196 & 19389 & -0.1 & 510 & 21801 & 18641 & -1.9 & 1377 & 22223 & 19183 \\
\hline Univ. Degree & 222 & 37296 & 37287 & -10.3 & 120 & 53772 & 74252 & 29.3 & 79 & 42418 & 48199 & 2.0 & 240 & 39198 & 35604 & -5.8 & 661 & 41590 & 47208 \\
\hline Missing & 17 & 18816 & 14459 & 34.7 & 17 & 14723 & 10910 & 5.4 & 26 & 9673 & 7135 & -30.8 & 24 & 14674 & 12971 & 5.0 & 84 & 13974 & 11665 \\
\hline
\end{tabular}

Legend: \% V = percentage variation of the mean $=100$ (mean - row total mean)/ (row total mean)

Table VII. Descriptive statistics on fiscal income in 2002 by occupation and by type of sample

\begin{tabular}{|c|c|c|c|c|c|c|c|c|c|c|c|c|c|c|c|c|c|c|c|}
\hline \multirow{2}{*}{ Occupation } & \multicolumn{4}{|c|}{ Interviewees } & \multicolumn{4}{|c|}{ Refusals } & \multicolumn{4}{|c|}{ Noncontacts } & \multicolumn{4}{|c|}{ Unused reserves } & \multicolumn{3}{|c|}{ Total } \\
\hline & $n$ & Mean & SD & $\% \mathrm{~V}$ & $n$ & Mean & SD & $\% \mathrm{~V}$ & $n$ & Mean & SD & $\% \mathrm{~V}$ & $n$ & Mean & SD & $\% \mathrm{~V}$ & $n$ & Mean & $\overline{S D}$ \\
\hline Entrepreneur & 53 & 48912 & 54932 & -1.8 & 44 & 78341 & 119303 & 57.2 & 32 & 49395 & 68648 & -0.9 & 119 & 39813 & 41691 & -20.1 & 248 & 49830 & 68763 \\
\hline Manager & 149 & 35389 & 27768 & 1.3 & 65 & 39167 & 34153 & 12.2 & 58 & 32215 & 23844 & -7.7 & 191 & 33928 & 28074 & -2.8 & 463 & 34919 & 28404 \\
\hline Dependent HSW & 160 & 22710 & 10097 & -1.4 & 128 & 23120 & 14514 & 0.4 & 101 & 23179 & 17769 & 0.6 & 216 & 23153 & 14710 & 0.5 & 605 & 23033 & 14159 \\
\hline Dependent MLSW & 177 & 18951 & 9374 & -0.2 & 160 & 20437 & 11223 & 7.6 & 120 & 18005 & 10301 & -5.2 & 338 & 18668 & 13304 & -1.7 & 795 & 18987 & 11677 \\
\hline Independent HSW & 37 & 31151 & 37912 & 12.8 & 15 & 22966 & 27642 & -16.8 & 15 & 33856 & 23640 & 22.6 & 45 & 24164 & 22148 & -12.5 & 112 & 27610 & 29063 \\
\hline OIW & 41 & 27121 & 24753 & 40.4 & 52 & 20890 & 17272 & 8.2 & 45 & 16640 & 16508 & -13.9 & 114 & 16846 & 13317 & -12.8 & 252 & 19315 & 17332 \\
\hline Retiree & 323 & 19460 & 18753 & 16.9 & 518 & 15103 & 13490 & -9.3 & 163 & 16720 & 15604 & 0.4 & 300 & 16269 & 11029 & -2.3 & 1304 & 16653 & 14840 \\
\hline Housewife \& Other & 135 & 9393 & 13557 & -2.7 & 193 & 11120 & 23094 & 15.2 & 100 & 10341 & 10945 & 7.1 & 175 & 7846 & 9995 & -18.7 & 603 & 9654 & 16166 \\
\hline Missing & 23 & 17295 & 13656 & 26.0 & 18 & 14008 & 11011 & 2.1 & 28 & 10398 & 8556 & -24.2 & 29 & 13930 & 12356 & 1.5 & 98 & 13725 & 11586 \\
\hline
\end{tabular}

Legend: HSW = Highly-Skilled Worker; MLSW = Medium-to-Low Skilled Workers, OIW = Other Independent Workers, \%V = 100 (mean - row total mean)/ (row total mean). 
The analysis of residuals of the regression model for the original FI showed that they were not normally distributed and presented a strong right asymmetry and heteroscedasticity. The transformation of FI through the natural logarithm, $\ln (Y)$, lost 14 observations: 7 because FI was negative and 7 because FI was equal to zero. It weakened, but did not eliminate, the heteroscedasticity and the residuals of regression model showed a strong left asymmetry. Therefore, the dependent variable, $Y$, was transformed through a logistic function

$$
\tilde{Y}=\frac{100}{1+\exp \left(-Y / Y_{0.80}\right)}
$$

where $\tilde{Y}$ assumed values between 0 and 100 and $Y_{0.80}$ was the $80^{\text {th }}$ percentile of $Y$ and represented a parameter that had to be chosen so that the distribution of residuals would be more consistent with the assumptions of the regression model. However, in the logistic transformation the impact of the covariates on the original dependent variable, $Y$, was not easily noticeable for continuous covariates and not always computable. For example, let $\Delta Y_{k}$ be the variation of $Y$ obtained by a change of one unit in the $k$-th covariate, $X_{k}$, holding the other covariates constant and equal to zero. Then, it will result that

$$
\Delta Y_{k}=-Y_{0.80} \ln \left[\left(\frac{100-\beta_{0}-x_{k} \beta_{k}-\beta_{k}}{100-\beta_{0}-x_{k} \beta_{k}}\right)\left(\frac{\beta_{0}+x_{k} \beta_{k}}{\beta_{0}+x_{k} \beta_{k}+\beta_{k}}\right)\right],
$$

where $\beta_{0}$ is the intercept of the model, $\beta_{k}$ is the estimated regression coefficient of $X_{k}$, while $x_{k}$ is the level of the covariate to which 1 is added, $\ln (\cdot)$ is the natural logarithm, which imposes that its argument should be positive. Therefore, the variation will depend on the level of $X_{k}$. For a dichotomous variable, the previous equation becomes

$$
\Delta Y_{k}=-Y_{0.80} \ln \left[\left(\frac{100-\beta_{0}-\beta_{k}}{100-\beta_{0}}\right)\left(\frac{\beta_{0}}{\beta_{0}+\beta_{k}}\right)\right] .
$$

A weak heteroscedasticity also remained in the case of the logistic transformation of FI. Therefore, parameter estimations were carried out using the robust Huber/White/sandwich estimator of variance in place of traditional calculation (Greene 2003). The results are reported in Table VIII, where there is a column displaying the impact on the untransformed dependent variable. For the sake of brevity, the reported estimations refer to the union of the four samples (interviewees, refusals, noncontacts, unused reserves), where the latter were distinguished through dummy variables. 
However, the estimation of one model for each sample did not show any substantial difference with respect to the objectives of the analysis.

The relationships of FI with gender, age, and education level proved to be highly significant and stable, as they continued to be valid also in each sample. On the average, men declared $4228.36 €$ more than women; individuals only with an elementary school level of education declared $3943.91 €$ less than those with a middle school education, while individuals with a secondary school diploma or university degree declared, respectively, 3662.78 and $10325.37 €$ more than those with a middle school education level. Age showed a non-linear trend, as expected: FI increased as age increased up to a given value, over which FI decreased with age. As for occupation, only the retirees proved to have a stable significant coefficient greater than zero. The effects of other modalities of the variables changed from one sample to another. However, in general, individuals with a long-term contract, working full-time, and having a paid job provided a FI greater than others. The occupation modalities (reported in Table VII) did not prove to be statistically significant in the total sample, except in the case of retirees, whose mean presented a positive difference equal to $1614.17 €$, with respect to the FI means of the other categories. In the total sample, the economic sector modalities "public administration" and "school" had negative coefficients, implying a lower FI compared to other sectors. However, the pattern of the relationships with the economic sector proved to be sensitive to the type of sample. Finally, the coefficient for the dummy variable denoting the sample of interviewees was positive with a borderline significance, implying a positive bias equal to $607.94 €$, i.e., holding constant the available covariates, the interviewed sample provided an overestimation equal to $607.94 €$. 
Table VIII. Parameter estimates for the regression model using the logistic transformation of fiscal income in 2002

\begin{tabular}{|c|c|c|c|c|c|}
\hline Independent variables & Coeff. & $\mathrm{SE}$ & $\mathrm{t}$ & $\mathrm{P}>\mathrm{t}$ & Impact* \\
\hline Man & 3.888 & 0.260 & 14.940 & 0.000 & 4228.36 \\
\hline Age/10 & 5.585 & 0.419 & 13.340 & 0.000 & 6043.47 \\
\hline$(\text { Age } / 10)^{\wedge} 2$ & -0.346 & 0.037 & -9.310 & 0.000 & -382.02 \\
\hline Elementary School & -3.514 & 0.363 & -9.680 & 0.000 & -3943.91 \\
\hline Secondary School & 3.363 & 0.346 & 9.710 & 0.000 & 3662.78 \\
\hline University Degree & 9.625 & 0.566 & 16.990 & 0.000 & 10325.37 \\
\hline Entrepreneur & 1.935 & 1.123 & 1.720 & 0.085 & 2117.92 \\
\hline Highly Skilled Worker & -0.754 & 0.496 & -1.520 & 0.128 & -834.34 \\
\hline Medium-Low Skilled Worker & -0.790 & 0.454 & -1.740 & 0.082 & -874.98 \\
\hline Retired Worker & 1.472 & 0.423 & 3.480 & 0.001 & 1614.17 \\
\hline Full-Time & 4.890 & 0.436 & 11.210 & 0.000 & 5301.76 \\
\hline Paid Worker & 5.396 & 1.316 & 4.100 & 0.000 & 5841.91 \\
\hline Non-Paid Worker & -1.026 & 0.771 & -1.330 & 0.183 & -1137.05 \\
\hline Long-Term Contract & 4.018 & 0.443 & 9.070 & 0.000 & 4367.42 \\
\hline Industry & 2.438 & 0.471 & 5.180 & 0.000 & 2664.10 \\
\hline Public Administration & -1.926 & 0.656 & -2.940 & 0.003 & -2144.43 \\
\hline School & -2.190 & 0.660 & -3.320 & 0.001 & -2441.41 \\
\hline Health-care & 2.728 & 0.748 & 3.650 & 0.000 & 2978.31 \\
\hline Services & 1.560 & 0.520 & 3.000 & 0.003 & 1710.06 \\
\hline Interviewees & 0.552 & 0.288 & 1.920 & 0.055 & 607.94 \\
\hline Constant & 39.687 & 1.065 & 37.270 & 0.000 & 46642.03 \\
\hline $\mathrm{R}^{\wedge} 2 /$ no. of cases & 0.425 & 4480 & & & \\
\hline
\end{tabular}

* The impact is expressed in the original value of the fiscal income

\subsection{FACTORS DETERMINING PARTICIPATION IN THE SURVEY}

The sample of unused reserves was assumed as the reference group and three data sets were set up combining the sample of unused reserves with one of the other samples: interviewees, refusals, and noncontacts. In each data set, a binary variable distinguished the two samples assuming a value equal to zero for the sample of unused reserves and equal to one for the other sample in the data set. Each data set was processed separately through a logistic model, where the dependent variable was the binary variable distinguishing the two samples. The initial set of covariates was the same as in the previous model, where the three dummy variables for the samples were substituted by FI, divided by 100000 to reduce its range. The logarithm transformation of FI did not improve the model and induced a reduction in the number of cases. The identification of 
significant covariates was carried out through the manual elimination of the nonsignificant covariates, but FI was always included in the model, as reported in Table IX. In these three logistic models, a positive coefficient for a given covariate implies that an increase in this covariate yields an increase in the probability of belonging to the sample added to the sample unused reserves; specifically, the interviewee sample for the first model, the refusal sample for the second model, and the noncontact sample for the third model. A negative coefficient implies the opposite meaning, that is, a decrease in that probability.

Table IX. Coefficients, standard errors (SE), and p-values (P) of logistic regressions

\begin{tabular}{|c|c|c|c|c|c|c|c|c|c|}
\hline \multirow{2}{*}{$\begin{array}{l}\text { Independent } \\
\text { variables }\end{array}$} & \multicolumn{3}{|c|}{ Interviewees/ U.R. } & \multicolumn{3}{|c|}{ Refusals/ U.R. } & \multicolumn{3}{|c|}{ Noncontacts/ U.R. } \\
\hline & Coeff. & $\mathrm{SE}$ & $\mathrm{P}>\mathrm{Z}$ & Coeff. & SE & $\mathrm{P}>\mathrm{Z}$ & Coeff. & SE & $\mathrm{P}>\mathrm{Z}$ \\
\hline FI/100000 & 0.339 & 0.210 & 0.106 & 0.504 & 0.178 & 0.005 & 0.222 & 0.242 & 0.358 \\
\hline Age/10 & 0.119 & 0.027 & 0.000 & 0.180 & 0.032 & 0.000 & & & \\
\hline$(\text { Age } / 10)^{\wedge} 2$ & & & & & & & 0.008 & 0.004 & 0.021 \\
\hline Man & -0.129 & 0.085 & 0.128 & & & & & & \\
\hline Elementary School & & & & 0.276 & 0.111 & 0.013 & 0.231 & 0.132 & 0.079 \\
\hline Secondary School & 0.306 & 0.100 & 0.002 & & & & & & \\
\hline University Degree & 0.600 & 0.146 & 0.000 & & & & & & \\
\hline Entrepreneur & -0.831 & 0.191 & 0.000 & -1.250 & 0.216 & 0.000 & -0.500 & 0.228 & 0.028 \\
\hline Manager & -0.330 & 0.148 & 0.025 & -1.116 & 0.174 & 0.000 & -0.453 & 0.175 & 0.009 \\
\hline Dependent HSW & & & & -0.403 & 0.147 & 0.006 & & & \\
\hline Dependent MLSW & -0.332 & 0.116 & 0.004 & -0.634 & 0.136 & 0.000 & -0.267 & 0.134 & 0.047 \\
\hline Independent HSW & & & & -1.016 & 0.315 & 0.001 & & & \\
\hline OIW & -0.681 & 0.195 & 0.000 & -0.863 & 0.192 & 0.000 & & & \\
\hline Retired worker & & & & & & & -0.311 & 0.154 & 0.044 \\
\hline Short-term contract & & & & & & & 0.352 & 0.204 & 0.084 \\
\hline Commerce & & & & 0.279 & 0.158 & 0.077 & -0.286 & 0.180 & 0.114 \\
\hline Health-care & & & & & & & -0.699 & 0.287 & 0.015 \\
\hline Services & -0.227 & 0.123 & 0.065 & & & & -0.286 & 0.146 & 0.051 \\
\hline Constant & -0.909 & 0.179 & 0.000 & -0.998 & 0.190 & 0.000 & -0.894 & 0.114 & 0.000 \\
\hline PseudoR $^{\wedge} 2 / n$ & 0.029 & 2625 & & 0.083 & 2720 & & 0.020 & 2189 & \\
\hline
\end{tabular}

Legend: HSW = Highly-Skilled Worker; MLSW = Medium-to-Low Skilled Workers; OIW = Other Independent Workers.

The probability of participation in the survey increased as FI and age increased, but FI showed only a borderline level of significance. It also increased among individuals with secondary school and university degrees. Entrepreneurs, managers, dependent medium-to-low skilled and other independent workers yielded negative coefficients, implying a decrease in the probability of participation in the survey. Individuals 
employed in the services economic sector yielded a negative coefficient with a borderline significance.

The probability of refusing the interview increased as FI and age increased. It also increased among individuals with an elementary school education level or with an occupation in the economic sector of commerce. Entrepreneurs, managers, dependent highly-skilled, dependent medium-to-low skilled, independent highly-skilled, and other independent workers yielded negative coefficients involving a decrease in the probability of refusing the interview.

The probability of noncontacts in the selected units varied nonlinearly with age. The coefficient of the squared term was positive implying that the probability decreased as age increased up to a certain value over which the probability of constituting noncontacts increased as age continued to increase, i.e., it was high for young and elderly people. In fact, young people are very mobile and rarely at home, while elders tend to not open the door to strangers or to interviewers ringing the doorbell. Individuals with an elementary school education level provided a positive coefficient, implying that they were less contactable than those with other education levels. The individuals with a shortterm contract also had a positive coefficient because they were generally young people and rarely at home. Surprisingly, entrepreneurs, managers, and dependent medium-to-low skilled workers yielded negative coefficients again, i.e., in all three data sets. Also, the retired workers proved to have negative coefficients, but often they were elderly people and, as mentioned above, they would not open the door to interviewers. Individuals working in the economic sectors of healthcare and services proved to have negative coefficients implying a decrease in the probability of not being contacted.

\subsection{FACTORS DETERMINING NONRESPONSE IN THE PANEL SAMPLE}

The survey on economic and social conditions of households in the Province of Modena was repeated in 2006 (ICESMO2) and in the city of Modena the proportion of overlaps in the two designs was fixed at about $1 / 3$ of the original sample constituted by 589 families in 2002. A proportion greater than $1 / 3$ of these families was drawn to obtain the panel sample in the event of many refusals to participate or many noncontacts: out of 319 families considered, 195 families were interviewed in the second wave of 2006. Therefore, it was possible to explore the behaviour of individuals following the previous process and the results proved to be very similar to those described above. The descriptive statistics regarding FI and the surveyed income in 2002 and 2006 are reported in Table X, but FI in 2002 was not directly comparable with the surveyed income in 2002 because, as indicated above, the reference periods differed and complex calculations were 
necessary to make them comparable. Some of these calculations are reported here only to provide some idea of their order of magnitude because a thorough discussion is beyond the scope of the paper. Therefore, the comments are limited to Figure 2 displaying the Epanechnikov kernel density estimates of FI, ranging from zero to 100,000€, to obtain a readable graph, for re-interviewed individuals, refusals, and noncontacts in the second wave of 2006. The density distributions showed long, heavy right tails, especially in the case of noncontacts, unlike in Figure 1, where the long right tail was that of the refusals. The distribution of participants had slightly shifted to the right with respect to the other two distributions, implying that high FIs were more easily surveyed than low FIs. The distribution of refusals showed a spike at about $18000 €$, which was higher than the modes of the distributions of the interviewees and noncontacts, involving a greater concentration of individuals with FIs of about $18000 €$ than among the interviewees or noncontacts.

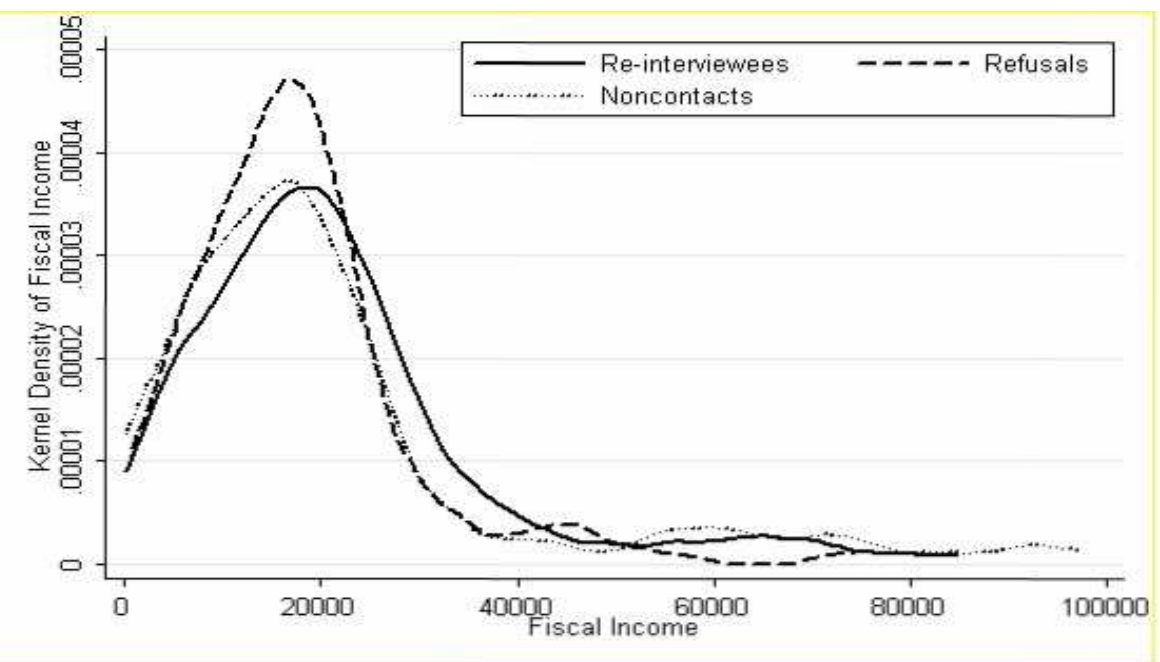

Figure 2. Epanechnikov kernel density estimates of fiscal income in 2002 for 2006 re-interviewees (second wave panel individuals), refusals, and noncontacts

When the weights determined in the survey carried out in 2002 were applied, then the distribution of FI for the individuals interviewed in the panel remained almost unchanged, while the distribution of refusals and noncontacts in the panel presented a reversed shape with the former being flattened and the latter attaining the greatest mode.

\section{Model selection via lasso methods}

The three logistic models, used to identify the factors affecting the different behaviours of the selected statistical units (interviewees, refusals, noncontacts), included only their marginal effect, but the covariates interacted presumably between them. For example, gender might interact with age and education level because the distribution of education 
level changed with age, as did the behaviour of individuals towards the interview. However, given the high number of factors involved in the explanation, only the introduction of the first-order interaction increased the number of covariates impressively. The first- and second-order interactions of the predictors listed above (see Section 4.1) generated a design matrix with 2626 columns, which was comparable to the sample size, and the standard GLM maximum likelihood algorithm was impracticable. In this context, the least absolute shrinkage and selection operator (Lasso) method was a useful tool for variable selection (Tibshirani 1996).

The Lasso, originally put forward in the context of linear regression models, has become a popular model selection tool also in the wider context of generalized linear models, such as logistic regression. The Lasso method maximises the usual log-likelihood function, which is subject to constraints regarding the sum of the absolute values of the coefficients. The geometrical nature of the constraints forces simultaneous variable selection and estimation. Namely, the estimation of the coefficients using the Lasso method are obtained by means of the following minimisation

$$
\hat{\beta}=\underset{\beta \in R^{p}}{\arg \min }\left(-\ell(\beta)+\lambda\|\beta\|_{1}\right),
$$

where $\ell(\beta)$ represents the log-likelihood of beta and $\|\cdot\|_{1}$ represents the $L_{1}$ norm. The constant $\lambda$ tunes the severity of the selection. Large values of $\lambda$ result in several regression parameters exactly equal to zero. Conversely, if $\lambda$ is small, no selection occurs. One advantage of Lasso selection is the stability of the signs and magnitude of the coefficients throughout the selection. This is not the case with the step-wise application of information-based criteria, such as AIC (Akaike Information Criterion).

Despite the appealing properties of Lasso, it should be noted that when both continuous and categorical predictors (factors) are present, Lasso selects individual dummy variables instead of whole factors. To overcome these difficulties, Meier, van de Geer, and Peter Bühlmann (2008) introduced the group Lasso for logistic regression by suitably modifying the penalty. Although the R package grplasso (Meier 2009) is the only package available to compute logistic grouped Lasso, it is still under development and its use is discouraged when dealing with large design matrices.

On the other hand, many established packages to compute logistic regression with plain Lasso penalty for the $R$ environment ( $R$ Development Core Team 2009) are available, such as the fastest and robust package glmnet (Friedman et al. 2009). The coordinate descendent algorithm of glmnet can handle large sparse matrices of thousands of predictors. 
The glmnet algorithm was applied to the three models described in Section 4.1. Interestingly, for all response variables, for the smallest value of $\lambda$ for which the algorithm converged, we obtained about 400 non-zero betas from 2626 initial predictors and the pseudo- $R^{2}$ increased up to 0.35 . Lasso selection appeared to enhance the models with substantially improved prediction performance. Certainly, an improved model can help researchers in future sample designs. Yet, interpreting 400 parameters is a challenging task.

A stronger dimensional reduction occurred when the tuning parameter was chosen by AIC minimization. Table XI reports the selected betas for the three models estimated via logistic Lasso. For the sake of brevity, it is noted briefly that the pseudo$R^{2}$ increased in all the three models, but that the number of covariates generally increased as well. There were many significant and interesting interactions. As expected, gender proved to be significant in the models only through the interaction with age, education level, and other covariates. The FI was important for refusals and noncontacts through interactions with some occupation modalities and other covariates.

\section{Conclusions}

The findings can, with due caution, be considered similar to those reported by other authors concerning the marginal effects of covariates affecting survey participation. The selective participation of sampling units yielded a positive bias of about $600 €$ for the FI of interviewees, holding constant the other factors. In fact, the distribution of FI proved to differ among the refusals, noncontacts, and interviewees and progressively shifted on the right implying that for low values of FI, the frequencies of refusals were higher than those of noncontacts, and the frequencies of noncontacts were higher than those of interviewees. Notwithstanding this, refusals presented a heavier and longer right tail than that of noncontacts and the right tail of noncontacts was heavier and longer than that of interviewees.

The probability of accepting to be interviewed increased by FI and age and for individuals with elementary or secondary school education levels. It decreased for some occupation modalities.

The probability of refusing the interview increased as FI and age increased, among individuals with an elementary school education level or with an occupation in the economic sector of commerce, while it decreased for some occupation modalities.

The probability of not being contacted followed a parabolic trend with age, implying that it was high for young and elderly people. It was also high for individuals with an elementary school education level and individuals with a short-term contract. It 
was low for entrepreneurs, managers, dependent medium-to-low skilled workers, retired workers, and individuals working in the economic sectors of healthcare and services.

The Lasso method made it relatively easy to introduce interactions between covariates, fixed up to the third order, and to select a reduced set of covariates. It was found that gender entered in the model through the interaction with age, education level, and other covariates. Also age and FI presented a complex pattern. Therefore, the interpretation of the results is not immediate. The method could be refined and improved to develop a user-friendlier version of the procedure. 
Table X. Descriptive statistics on fiscal income and equivalent surveyed income in 2002 and 2006 for the 2002-2006 panel

\begin{tabular}{|c|c|c|c|c|c|c|c|c|c|}
\hline \multirow{2}{*}{$\begin{array}{l}\text { Type of panel sample } \\
\text { from the } 1^{\text {st }} \text { to the } 2^{\text {nd }} \text { wave }\end{array}$} & \multicolumn{3}{|c|}{ Fiscal Income 2002} & \multicolumn{3}{|c|}{ Equivalent Income 2002} & \multicolumn{3}{|c|}{ Equivalent Income 2006} \\
\hline & $n$ & Mean & SD & $n$ & Mean & $\mathrm{SD}$ & $n$ & Mean & SD \\
\hline Re-interviewed in $2^{\text {nd }}$ wave & 418 & 18987.3 & 24031.2 & 423 & 28264.2 & 15876.1 & 423 & 32461.7 & 17097.2 \\
\hline Refusals in the $2^{\text {nd }}$ wave & 131 & 16944.8 & 18629.6 & 149 & 25145.8 & 17897.9 & 0 & & \\
\hline Noncontacts in the $2^{\text {nd }}$ wave & 84 & 19851.2 & 23350.7 & 102 & 25222.5 & 14468.3 & 0 & & \\
\hline Total & 633 & 18679.2 & 22910.8 & 674 & 27114.5 & 16190.7 & 423 & 32461.7 & 17097.2 \\
\hline
\end{tabular}

Table XI. Coefficients, standard errors (SE), and p-values (P) of logistic regressions with interactions

\begin{tabular}{|c|c|c|c|c|c|c|c|c|c|}
\hline \multirow[t]{2}{*}{ Independent variables } & \multicolumn{3}{|c|}{ Interviewees/ Reserves } & \multicolumn{3}{|c|}{ Refusals/ Reserves } & \multicolumn{3}{|c|}{ Noncontacts/ Reserves } \\
\hline & Coeff. & SE & $\mathrm{P}>\mathrm{Z}$ & Coeff. & SE & $\mathrm{P}>\mathrm{Z}$ & Coeff. & $\mathrm{SE}$ & $\mathrm{P}>\mathrm{Z}$ \\
\hline Man * DMLSW & & & & -0.666 & 0.242 & 0.006 & & & \\
\hline Man $*$ Age $1 *$ Retiree & -0.048 & 0.021 & 0.025 & -0.060 & 0.024 & 0.012 & -0.107 & 0.029 & 0.000 \\
\hline Man * Degree * DMLSW & 3.221 & 1.483 & 0.030 & 2.711 & 1.335 & 0.042 & & & \\
\hline Man $*$ Full-time $*$ Short-term contract & & & & & & & 1.643 & 0.640 & 0.010 \\
\hline Man * Industry * DHSW & 0.630 & 0.312 & 0.044 & & & & & & \\
\hline Man $*$ Services $*$ Manager & -1.396 & 0.714 & 0.051 & & & & & & \\
\hline Age $1=$ Age $/ 10$ & 0.394 & 0.148 & 0.008 & 0.263 & 0.146 & 0.073 & & & \\
\hline Age1 $*$ Elementary School & & & & -0.072 & 0.033 & 0.027 & & & \\
\hline Age1 $*$ Manager & & & & -0.487 & 0.288 & 0.091 & & & \\
\hline Age $1 *$ Full-time & -0.202 & 0.045 & 0.000 & & & & -0.105 & 0.050 & 0.034 \\
\hline Age1 $*$ Entrepreneur * Industry & & & & & & & -0.435 & 0.200 & 0.030 \\
\hline University Degree & & & & 0.422 & 0.148 & 0.004 & & & \\
\hline Elementary School $*$ Retiree & 0.672 & 0.269 & 0.012 & 0.647 & 0.291 & 0.026 & & & \\
\hline Elementary School $*$ Part-time $*$ Commerce & & & & & & & 2.494 & 1.174 & 0.034 \\
\hline Secondary School $*$ Part-time $*$ DHSW & & & & & & & 1.294 & 0.603 & 0.032 \\
\hline
\end{tabular}


(continued)

Table XI. Coefficients, standard errors (SE), and p-values (P) of logistic regressions with interactions

\begin{tabular}{|c|c|c|c|c|c|c|c|c|c|}
\hline \multirow[t]{2}{*}{ Independent variables } & \multicolumn{3}{|c|}{ Interviewees/ Reserves } & \multicolumn{3}{|c|}{ Refusals/ Reserves } & \multicolumn{3}{|c|}{ Noncontacts/ Reserves } \\
\hline & Coeff. & $\mathrm{SE}$ & $\mathrm{P}>\mathrm{Z}$ & Coeff. & SE & $\mathrm{P}>\mathrm{Z}$ & Coeff. & SE & $\mathrm{P}>\mathrm{Z}$ \\
\hline University Degree * Short-term contract * Manager & & & & 3.123 & 1.319 & 0.018 & & & \\
\hline Entrepreneur & & & & -0.598 & 0.284 & 0.035 & 0.571 & 0.328 & 0.082 \\
\hline Full-time $*$ OIW & & & & -0.794 & 0.315 & 0.012 & & & \\
\hline Full-time $*$ Services $*$ Paid worker & & & & & & & -1.320 & 0.596 & 0.027 \\
\hline Full-time * Services $*$ DMLSW & & & & & & & -1.823 & 0.631 & 0.004 \\
\hline Part-time & -1.069 & 0.330 & 0.001 & & & & -0.642 & 0.370 & 0.083 \\
\hline Part-time * Industry * GES-manager & & & & & & & 2.972 & 1.337 & 0.026 \\
\hline Part-time * School $*$ DMLSW & & & & & & & 1.642 & 0.856 & 0.055 \\
\hline Part-time $*$ School $*$ Short-term contract & & & & 2.701 & 1.356 & 0.046 & & & \\
\hline Health-care & & & & & & & -1.378 & 0.455 & 0.002 \\
\hline Services * DMLSW & & & & & & & 0.903 & 0.478 & 0.059 \\
\hline Industry $*$ OIW $*$ Non-paid worker & & & & 1.072 & 0.566 & 0.058 & 1.119 & 0.553 & 0.043 \\
\hline ES-health $*$ Manager $*$ Long-term contract & 1.146 & 0.546 & 0.036 & 1.030 & 0.461 & 0.025 & 2.365 & 0.676 & 0.000 \\
\hline FI $*$ Retiree & & & & 1.587 & 0.690 & 0.021 & & & \\
\hline FI $*$ DHSW $*$ Short-term contract & & & & & & & -11.61 & 5.063 & 0.022 \\
\hline FI $*$ DHSW $*$ Public administration & & & & & & & 4.642 & 2.052 & 0.024 \\
\hline Constant & -1.608 & 0.362 & 0.000 & -1.153 & 0.347 & 0.001 & -0.763 & 0.392 & 0.052 \\
\hline PseudoR^2/ $n$ & 0.051 & 2625 & & 0.105 & 2720 & & 0.087 & 2189 & \\
\hline
\end{tabular}

Legend: DHSW = Dependent Highly-Skilled Workers; DMLSW = Dependent Medium-to-Low Skilled Workers; OIW = Other Independent Workers 


\section{References}

Baldini, M., Bosi, P. \& Silvestri, P. (eds.) (2004). La ricchezza dell'equità. Distribuzione del reddito e condizioni di vita in un'area a elevato benessere. Bologna: il Mulino.

Banca d'Italia (2002). I bilanci delle famiglie italiane nell'anno 2000. Supplementi al bollettino statistico, anno XII, no. 6. Roma: Banca d'Italia.

Banca d'Italia (2008). I bilanci delle famiglie italiane nell'anno 2006. Supplementi al bollettino statistico, anno XVIII, no. 7. Roma: Banca d'Italia.

Biancotti, C., D’Alessio, G. \& Neri, A. (2004). Errori di misura nell'indagine sui bilanci delle famiglie italiane. Temi di discussione, no. 520. Roma: Banca d'Italia.

Biemer, P. P. \& Lyberg, L. E. (2003). Introduction to Survey Quality. New York: John Wiley \& Sons.

Bound, J., Brown, C. \& Mathiowetz, N. (2001). Measurement Error in Survey Data. In: J. J. Heckman \& E. Leamer (eds.), Handbook of Econometrics, vol. 5. Amsterdam: Elsevier, pp. 3705-3843.

Brancato, G., Carbini, R., Pellegrini, C., Signore, M. \& Simeoni, G. (2006). Assessing Quality through the Collection and Analysis of Standard Quality Indicators: The ISTAT Experience, Proceedings of Q2006, European Conference on Quality in Survey Statistics, www.oecd.org/dataoecd/34/37/37278499.pdf. Accessed 23 August 2007

Campanelli, P. \& O’Muircheartaigh, C. (1999). Interviewers, Interviewer Continuity, and Panel Survey Nonresponse. Quality \& Quantity 33 (1): 59-76.

Cannari, L., \& D’Alessio, G. (1992). Mancate interviste e distorsione degli stimatori. Temi di discussione, no. 172. Roma: Banca d'Italia.

Cohen, S. B. \& Carlson, B. L. (1995). Characteristics of reluctant respondents in the national medical expenditure survey. Journal of Economic and Social Measurement 21: 269-296.

Collier, J. E. (2007). An analysis of how nonresponse error is assessed in academic marketing research. Marketing Theory 7 (2): 163-183.

Couper, M. P. \& Groves, R. M. (1992). The Role of Interviewer in Survey Participation. Survey Methodology 18: 263-278.

Curtin, R. T., Juster, F. T. \& Morgan, J. N. (1989). Survey Estimates of Wealth: An assessment of Quality. In: R. E. Lipsey \& H. S. Tice (eds.), The Measurement of Saving, Investment, and Wealth, National Bureau of Economic Research, Studies in Income and Wealth, vol. 52. Chicago, IL: The University of Chicago Press, pp. 473-548.

D'Alessio, G. \& Faiella, I. (2002). Non-response Behaviour in the Bank of Italy's Survey of Household Income and Wealth, Temi di discussione, no. 462. Roma: Banca d'Italia.

de Bruwer, W. J. (1995). A Sampling Methodology and Replacement Procedure for Reducing Nonresponse Bias in Fixed-Address Industrial Surveys. Journal for Studies in Economics and Econometrics 19 (2): 61-75.

de Heer, W. (1999). International response trends: results of an international survey. Journal of Official Statistics 15(2): 129-142.

de Leeuw, E. \& de Heer,W. (2002). Trends in household survey nonresponse: a longitudinal and international comparison. In: Groves, R. M., Dillman, D. A., Eltinge, J. L., Little, R. J. A. (eds.), Survey Nonresponse, pp. 41-54. Wiley, New York .

Delener, N. (1995). An Integrative Review of Nonresponse Errors in Survey Research: Major Influences and Strategies. Research in marketing 12: 49-80.

Franses, P. H., Geluk, I. \& Van Homelen, P. (1999). Modeling Item Nonresponse in Questionnaires. Quality \& Quantity 33 (2): 203-213.

Friedman, J., Hastie, T. \& Tibshirani, R. (2009). glmnet: Lasso and elastic-net regularized generalized linear models. $\mathrm{R}$ package version 1.1-3. http://wwwstat.stanford.edu/ hastie/Papers/glmnet.pdf. Accessed 10 May 2009

Gallo, F., Mastrovita, S., Siciliani, I. \& Arcieri, G.B. (2004). Il processo di produzione dell'Indagine ECHP. In: G. Brancato (ed.), Note Metodologiche dell'Annuario Statistico Italiano, No. 4.4 Roma: Istat. http://www.istat.it/dati/pubbsci/documenti/Documenti/doc_2004/ 2004_4.doc. Accessed 23 August 2007

Goyder, J. (1987). The Silent Minority: Nonresponse in Household Interview Survey. Cambridge: Polity Press.

Greene, W. H. (2003). Econometric Analysis, fifth edition. Upper Saddle River, NJ: Prentice Hall.

Hox, J. \& de Leeuw, E. (2002). The Influence of Interviewers' Attitude and Behavior on Household Survey Nonresponse: An International Comparison. In: R. M. Groves, D. A. Dillman, J. L. Eltinge \& R. J. A. Little (eds.), Survey Nonresponse. New York: Wiley \& Sons, pp. 103-120. 
Hüfken, V. (2009). Supplementary questionnaire and nonresponse-results from the German ISSP survey. Quality \& Quantity DOI 10.1007/s11135-009-9221-3

Hurd, M., Juster, F. T. \& Smith, J. P. (2003). Enhancing the Quality of Data on Income. Journal of Human Resources 38 (3): 758-772.

Juster, F. T. \& Smith, J. P. (1997). Improving the Quality of Economic Data: Lessons from HRS and AHEAD. Journal of the American Statistical Association 92: 1268-78.

Kupek, E. (1998). Determinants of Item Nonresponse in a Large National Sex Survey. Archives of Sexual Behavior 27 (6): 581-594.

Lessler, J. T. \& Kalsbeek, W. D. (1992). Nonsampling Errors in Surveys. New York : Wiley \& Sons.

Levy, P. S. \& Lemeshow, S. (1991). Sampling of Populations: Methods and Applications. New York: John Wiley \& Sons.

Little, R. J. A. \& Rubin, D. B. (1987). Statistical Analysis with Missing Data. New York: John Wiley \& Sons.

Meier, L., van de Geer, S. \& Bühlmann, P. (2008). The group lasso for logistic regression. Journal of the Royal Statistical Society, Series B 70 (1): 53-71

Meier, L. (2009). grplasso: Fitting user specified models with Group Lasso penalty. R package version 0.4-2. http://CRAN.R-project.org/package=grplasso. Accessed 10 May 2009

Moore, J. C., Stinson, L. L. \& Welniak, E. J. Jr. (2000). Income measurement error in surveys: a review. Journal of Official Statistics 16(4): 331-361.

Quintano, C. \& Lucev, D. (1990). Le mancate risposte in esperienze di indagini reddituali. Quaderni sardi di economia 20 (3): 253-278.

R Development Core Team (2009). R: A language and environment for statistical computing. Vienna, Austria: R Foundation for Statistical Computing. ISBN 3-900051-07-0, URL http://www.R-project.org. Accessed 10 May 2009

Rubin D. B. (1988). Multiple Imputation for Nonresponse in Surveys. New York: John Wiley \& Sons.

Särndal, C.-E., Swensson, B. \& Wretman, J. (1992). Model Assisted Survey Sampling. Berlin: Springer-Verlag.

Särndal, C.-E. \& Lundström, S. (2005). Estimation in Surveys with Nonresponse. New York: Wiley.

Schräpler, J.P. (2006). Explaining Income Nonresponse - A Case Study by means of the British Household Panel Study (BHPS). Quality \& Quantity 40 (6): 1013-1036.

Stocké, Volker (2006). Attitudes Toward Surveys, Attitude Accessibility and the Effect on Respondents' Susceptibility to Nonresponse. Quality \& Quantity 40 (2): 259-288.

Tibshirani, R. (1996). Regression shrinkage and selection via the lasso. Journal of the Royal Statistical Society, Series B 58 (1): 267-288.

Tourangeau, R., Rips, L. J. \& Rasinki, K. (2000). The Psychology of Survey Response. Cambridge: Cambridge University Press.

Tu, S.-H. \& Liao, P.-S. (2007). Social Distance, Respondent Cooperation and Item Nonresponse in Sex Survey. Quality \& Quantity 41:177-199. 


\section{University Library}

\section{- M M N E R VA A gateway to Melbourne's research publications}

Minerva Access is the Institutional Repository of The University of Melbourne

Author/s:

Lalla, M;Ferrari, D;Frederic, $P$

Title:

Unit nonresponse errors in income surveys: A case study

Date:

2012-10-01

Citation:

Lalla, M., Ferrari, D. \& Frederic, P. (2012). Unit nonresponse errors in income surveys: A case study. Quality and Quantity, 46 (6), pp.1769-1794. https://doi.org/10.1007/ s11135-011-9557-3.

Persistent Link:

http://hdl.handle.net/11343/282561 